\title{
Spinal Arteriovenous Metameric Syndrome: Clinical Manifestations and Endovascular Management
}

\author{
Y. Niimi, N. Uchiyama, L. Elijovich, and A. Berenstein
}

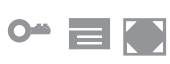

\begin{abstract}
BACKGROUND AND PURPOSE: SAMS is a rare form of SCAVM. We discuss the clinical presentation, endovascular management, and outcome of this disease in our series.
\end{abstract}

MATERIALS AND METHODS: Retrospective review was performed in patients with SCAVM and SAMS who underwent angiography with intent to treat at our institution from 1980 to 2010.

RESULTS: One hundred forty-eight SCAVMs were identified, and 28 (19\%) of these were SAMS. Of these 28 patients, 24 had nidus-type AVMs and 4 had fistulas. SAMS were more prevalent in females (71\% versus $48 \%$ ), and also presented earlier than non-SAMS SCAVMs. Intradural hemorrhage (SAH or hematomyelia) was the most common presentation and more common than in non-SAMS lesions. Twenty-six patients underwent embolization of the intradural lesion in 50 sessions. Thirteen patients underwent treatment of intradural aneurysms in 16 sessions. Six patients underwent embolization of extradural lesions in 16 sessions. Twenty-three patients had an average of 94 months of clinical follow-up (3-309 months) after the first treatment, during which 5 patients had hemorrhages. Angiographic follow-up was performed in 20 patients at an average of 85 months (range, 3-309 months), which showed new development or enlargement of an aneurysm in 5 patients. This type of angiographic progression was more common in patients with SAMS.

CONCLUSIONS: SAMS most commonly presents with hemorrhage from the SCAVM. Endovascular embolization can be performed safely with good functional outcome, though most patients clinically deteriorate in the long term. Periodic angiographic follow-up with intent to perform preventive target embolization is important to control the disease.

ABBREVIATIONS: $\mathrm{AVF}=$ arteriovenous fistula; $n$-BCA $=\mathrm{N}$-butyl 2-cyanoacrylate; SAMS $=$ spinal arteriovenous metameric syndrome; SCAVF $=$ spinal cord arteriovenous fistula; SCAVM = spinal cord arteriovenous malformation

$\mathbf{T}$ he SCAVM is a rare disease that is associated with frequent hemorrhages and a variety of neurologic deficits. ${ }^{1}$ Among these is a subcategory of lesions that consists of multiple vascular malformations that affect $\geq 2$ tissues derived from the same spinal metameric segment. These lesions have been variably termed

Received March 21, 2012; accepted after revision May 10.

From the Center for Endovascular Surgery (Y.N., A.B.), Institute for Neurology and Neurosurgery, Roosevelt Hospital, New York, New York; Department of Neurosurgery (N.U.), Kanazawa University School of Medicine, Kanazawa, Japan; and Department of Neurology (L.E.), University of Tennessee, Memphis, Tennessee.

Paper previously presented in part at: 11th Congress of World Federation of Interventional and Therapeutic Neuroradiology, November 8-11, 2011; Capetown, South Africa.

Please address correspondence to Yasunari Niimi, Center for Endovascular Surgery, Institute for Neurology and Neurosurgery, Roosevelt Hospital, Suite 10G, New York, NY 10019; e-mail: yasuniimi@aol.com

- Indicates open access to non-subscribers at www.ajnr.org

$\equiv$ Indicates article with supplemental on-line tables.

4 Indicates article with supplemental on-line figures.

http://dx.doi.org/10.3174/ajnr.A3212
Cobb syndrome, extra-intradural, juvenile, and SAMS. ${ }^{2-4}$ We prefer the term SAMS because of its embryologic consideration. It can involve the spinal cord, bone, epidural space, paraspinal soft tissues or muscles, subcutaneous tissues, and skin. It can occur in any of 31 spinal segments and can involve $>1$ segment. $^{5}$

Whether the SCAVM component of SAMS is different from non-SAMS lesions in its clinical presentation, natural history, and vascular anatomy is unknown. In this series, we present our experience in the treatment of a consecutive cohort of patients with SAMS with both long-term angiographic and also clinical outcomes.

\section{MATERIALS AND METHODS}

The data base of SCAVMs at our institution from 1980 to 2010 was queried to identify all patients with SAMS who underwent spinal angiography with intent to treat. A retrospective review of the chart and imaging was then performed to analyze the clinical presentation, angioarchitecture, details of treatment, and followup. Long-term clinical results were characterized as symptomatic 
improvement, unchanged, or worsening. Follow-up was performed by telephone or office visits.

\section{Angiographic/Embolization Protocol}

All angiographic procedures were performed with the patients under general anesthesia with neuroleptic agents and neurophysiologic monitoring, including somatosensory evoked potentials and transcranial motor-evoked potentials. Superselective catheterization of the appropriate feeding pedicles was performed by using a microcatheter before embolization to analyze the vascular anatomy of the lesion. Pharmacologic provocative testing was performed with superselective injection of amobarbital sodium and lidocaine when indicated. The details of the indication, technique, and results of neurophysiologic monitoring and provocative testing for SCAVM have been described previously. ${ }^{6}$ Our treatment strategy is to perform endovascular embolization targeting the symptomatic lesion, which is most commonly the intradural AVM or AVF. We perform targeted partial embolization for nidus-type SCAVMs to close aneurysms and fistulas to prevent hemorrhage and improve spinal cord hemodynamics to treat symptomatic myelopathy/venous hypertension. Complete angiographic cure is not the goal with most intradural lesions with the exception of SCAVFs. $n$-BCA was the main embolic agent for both intra- and extradural lesions. Particulate embolization was used when $n$-BCA embolization was not feasible on the basis of the flow dynamics of the lesion. Coils were used for embolization of aneurysms, high-flow fistulas, and protection of the normal vascular territories when embolizations were carried out from proximal positions (nonintranidal). Ethylene-vinyl alcohol copolymer (Onyx; ev3, Irvine, California) was used exclusively for extradural lesions.

\section{RESULTS}

A total of 148 patients underwent spinal angiography with intent to treat for SCAVMs, including SCAVFs, among whom 28 patients (19\%) were found to have SAMS. These included 24 nidustype SCAVMs and 4 SCAVFs (1 single-hole and 3 multiple-hole AVFs). The SAMS group tended to have more nidus-type lesions $(24 / 28,85.7 \%)$ compared with the non-SAMS group, which had 80 nidus-type SCAVMs of 120 cases $(80 / 120,66.7 \%$; $P=.0649$, Fisher exact test). There were 8 males and 20 females, in contrast to the non-SAMS group, which consisted of 62 males and $58 \mathrm{fe-}$ males; there was significant female dominance in the SAMS group $(P<.0001$, Fisher exact test $)$. The average age of onset was $19.4 \pm$ 10.8 years of age, which was significantly younger compared with non-SAMS patients $(27.7 \pm 18$ years of age; $P=.0239, t$ test $)$. The average age at diagnosis tended to be younger in patients with SAMS $(26.4 \pm 16.3$ years $)$ than in non-SAMS patients $(30.3 \pm$ 18.1 years), but there was no statistical significance $(P=.5100, t$ test).

The associated metameric involvement was incomplete in most patients. Among 28 cases, associated lesions included 15 epidural, 13 paraspinal, 12 osseous, 11 radicular, 4 soft-tissue, 2 skin, and 1 with limb involvement. The lesions were almost evenly distributed from the cervicomedullary junction to the conus.

The most common presenting symptom was intradural hemorrhage, occurring in 18 patients (11 spinal SAHs and 7 hemato- myelias, 64\%). Seven patients (25\%) presented with nonhemorrhagic neurologic deficits, and 3 patients $(11 \%)$ presented with radicular pain without neurologic deficits (On-Line Table 1). Four additional patients developed intradural hemorrhage (1 spinal SAH and 3 hematomyelias) following nonhemorrhagic presentation before the first treatment. Overall, 22 patients (79\%) experienced hemorrhage before the first angiographic study with intent to treat. This was statistically significantly higher compared with non-SAMS patients in whom 70 of 120 (58\%) experienced hemorrhage before the first angiographic study with intent to treat $(P=.0173$, Fisher exact test). Multiple episodes of hemorrhage before the first angiographic study with intent to treat also occurred more often in the SAMS group (11 patients, 39\%) than in the non-SAMS group (26 patients, $22 \%$ ), but without statistical significance $(P=.0872$, Fisher exact test) (On-line Table 2$)$.

Five patients had received previous treatments, including 3 surgeries and 4 embolizations, for spinal cord lesions at an outside institution. An additional 2 patients had limb amputation, 1 for the limb AVM and the other for intractable pain. No patients underwent surgery after they were referred to us. In our institution, 26 patients underwent a total of 50 sessions of endovascular treatment for the intradural lesions, ranging from 1 to 6 sessions per patient. During these sessions of treatment, intradural aneurysms were treated in 16 sessions in 13 patients. Neurologic deterioration after the treatment occurred in 4 patients, including 1 permanent and 3 temporary deficits. Six patients underwent 16 sessions of embolization for symptomatic extradural lesions. Most of these lesions were extensive and required multiple sessions of treatment.

Twenty-three patients had clinical follow-up ranging from 3 months to 309 months (mean, 94 months) following the initial endovascular treatment. Five patients (22\%) had hemorrhage during follow-up. This was statistically significantly higher compared with the non-SAMS group $(P=.043$, Fisher exact test). In the non-SAMS group, 95 patients had clinical follow-up ranging from 1 to 232 months (mean, 45 months) following the initial endovascular treatment. Among them, 6 patients (6.3\%) had hemorrhage during the follow-up.

Nine patients had clinical follow-up of $<5$ years. Of these, 5 showed improvement; 3 , no change; and 1 experienced deterioration at the last follow-up in comparison with the evaluation before the initial treatment. Fourteen patients had $>5$ years of follow-up; 4 patients demonstrated improvement, 4 were unchanged, and 6 deteriorated during the follow-up period.

Angiographic follow-up beyond 30 days was performed in 20 patients. The range was 3-309 months (mean, 85 months). Seven patients developed new aneurysms or demonstrated enlargement of a pre-existing aneurysm. Two of these lesions were considered hemorrhage-related pseudoaneurysms and were excluded. The remaining 5 (25\%) were unrelated to hemorrhage or were discovered before a clinical hemorrhage. These 5 patients had a longterm angiographic follow-up ranging from 69 to 314 months. However, the time of last follow-up did not reflect the timing of aneurysmal development or enlargement. Some aneurysms were discovered earlier and treated, while others were conservatively followed. In comparison, 57 non-SAMS patients underwent follow-up angiography, ranging from 1 to 207 months (mean, 44 
months). Among the non-SAMS group, only 2 patients $(3.5 \%)$ showed new development or enlargement of an aneurysm. These patients had long-term angiographic follow-up at 85 months and 133 months, respectively. Patients with SAMS demonstrated a statistically significant angiographic progression of disease in regard to the enlargement or development of aneurysms $(P=.0112$, Fisher exact test).

\section{Representative Cases}

Case 1. A 19-year-old man presented with symptoms of cervical myelopathy and weakness of the right upper extremity (Fig 1). MR imaging and subsequent spinal angiography demonstrated SAMS. Two discrete nidi of the SCAVM at the C2-C3 and C5 vertebral levels were found as well as high-flow multilevel paravertebral and intraosseous fistulas from the $\mathrm{C} 2$ to $\mathrm{C} 4$ levels supplied by bilateral vertebral, dorsocervical, ascending cervical, and external carotid arteries. The first treatment focused on the paravertebral component, with venous drainage to the epidural veins, causing myelopathy due to spinal cord congestion or compression. He completely recovered from the right-sided weakness after the first $n$-BCA treatment. He underwent 3 subsequent $n$-BCA embolizations in the next 7 months due to recurrent right-sided weakness. These series of treatments stabilized his symptoms, and follow-up CT showed progressive filling of the osseous defects of the affected spine. Twenty months after the fourth treatment, CT of the spine demonstrated recurrent erosion of the spine (On-line Figure 1), though he remained asymptomatic. He then underwent 2 treatments of the paravertebral component with Onyx. The seventh treatment was provoked by an episode of acute left upper and lower extremity weakness, which developed 5 months after the last Onyx treatment. MR imaging at that time demonstrated increased T2 signal in the spinal cord and mass effect from an enlarging aneurysm. Embolization of the aneurysm through the anterior spinal artery with $n$-BCA resulted in dramatic improvement of symptoms. Two years later, he underwent another embolization of the paraspinal fistulas with Onyx due to recurrent right-sided weakness with subsequent improvement. He has been neurologically stable with minimal right-sided weakness for $>18$ months since the last treatment.

Case 2. A 39-year-old man initially presented with SAH at 21 years of age. No definitive diagnosis was made at that time (Fig 2). He presented, in delayed fashion, at 39 years of age with severe chronic back pain radiating to the right leg with atrophy. Angiography demonstrated AVMs of the lower spinal cord and the right nerve roots at L4, L5, and S2. He underwent 4 embolizations in 1995 and 1997 for the radicular AVMs with significant improvement of radicular pain. During angiography, a small aneurysm of the feeding anterior spinal artery was noted but was not treated. In 2004, he developed a second spinal SAH. Angiographic work-up demonstrated enlargement of the anterior spinal artery aneurysm with development of a pseudoaneurysm at the tip. He underwent coil embolization for the aneurysm. Follow-up angiography in 2007 demonstrated stable occlusion of the aneurysm and increased flow through the SCAVM. Attempted embolization was aborted because of a positive provocative test. In 2011, he underwent another spinal angiography due to an episode of radiating back pain and subsequent left lower extremity numbness. The study showed spontaneous occlusion of the anterior spinal artery near the level of the aneurysm, which was thought to be due to spontaneous dissection of the anterior spinal artery (Case 3, Online Figure 2).

\section{DISCUSSION}

The concept of SAMS is spinal extension of the craniofacial arteriovenous metameric syndrome proposed by Bhattacharya et al. ${ }^{4}$ This was based on research on avian embryos demonstrating that mesoderm and neural crest cells from a given metameric level occupy the same territory in the face and brain and that these 2 cell types cooperate in myogenesis and vasogenesis. ${ }^{7}$ During vasogenesis, endothelial cells derive from the mesoderm and the tunica media derives from the neural crest cells. Therefore, a genetic defect or somatic mutation in the neural crest or adjacent mesoderm before migration causes development of $>1$ vascular malformation with a metameric distribution. Therefore, the causative event creating a vascular malformation such as a genetic mutation occurred earlier in the case of SAMS than in a solitary SCAVM.

Rodesch et $\mathrm{al}^{8}$ proposed a classification of spinal cord AVMs, considering the timing of the causative event responsible for creation of a vascular malformation such as gene mutation. They classified SAMS under the category of "genetic nonhereditary" along with multimyelomeric AVMs and syndromic AVMs such as Klippel-Trenaunay-Weber syndrome and Parkes Weber syndrome. In their series of 155 cases of spinal cord arteriovenous shunting, $20.6 \%$ belonged to the category of "genetic nonhereditary." These consisted of $6.4 \%$ metameric, $11 \%$ multimyelomeric, and $3.2 \%$ syndromic cases. They classified cases of a SCAVM associated with a radicular AVM as "multimyelomeric," which should be classified as "metameric" if the AVMs are at the same metameric level as the authors discussed in the article. This would probably make SAMS approximately $12 \%$ of SCAVMs in their series, judging from the earlier report of the same group. In their series, SAMS showed male dominance by 2:1, which is the opposite of our cases in which there was strong female dominance by $5: 2 .^{2}$

Berenstein et $\mathrm{al}^{5}$ performed a literature search and found that metameric lesions were reported to be $7 \%-14 \%$ of SCAVMs. SAMS may be underestimated because associated lesions, especially radicular AVMs, are frequently missed or excluded from the metameric lesions. In our series, there were a significant number of radicular AVMs, which may account for the higher number of SAMS (19\%) compared with the report of Rodesch et al. ${ }^{8}$ The frequency of radicular AVMs should be noted in comparison with craniofacial arteriovenous metameric syndrome, in which cranial nerve AVMs are extremely rare.

Our series illustrates that most cases of SAMS have an intradural component but rarely manifest as a complete angiographic expression of the disease with involvement of all tissue elements that encompass the metamere. We experienced 1 additional patient who had cervical paraspinal and spinal AVMs associated with a muscle AVM at the same level without intradural malformation. This case can also be considered within a spectrum of SAMS.

The most common presenting symptom was spinal $\mathrm{SAH}$

AJNR Am J Neuroradiol 34:457-63 Feb 2013 www.ajnr.org 459 

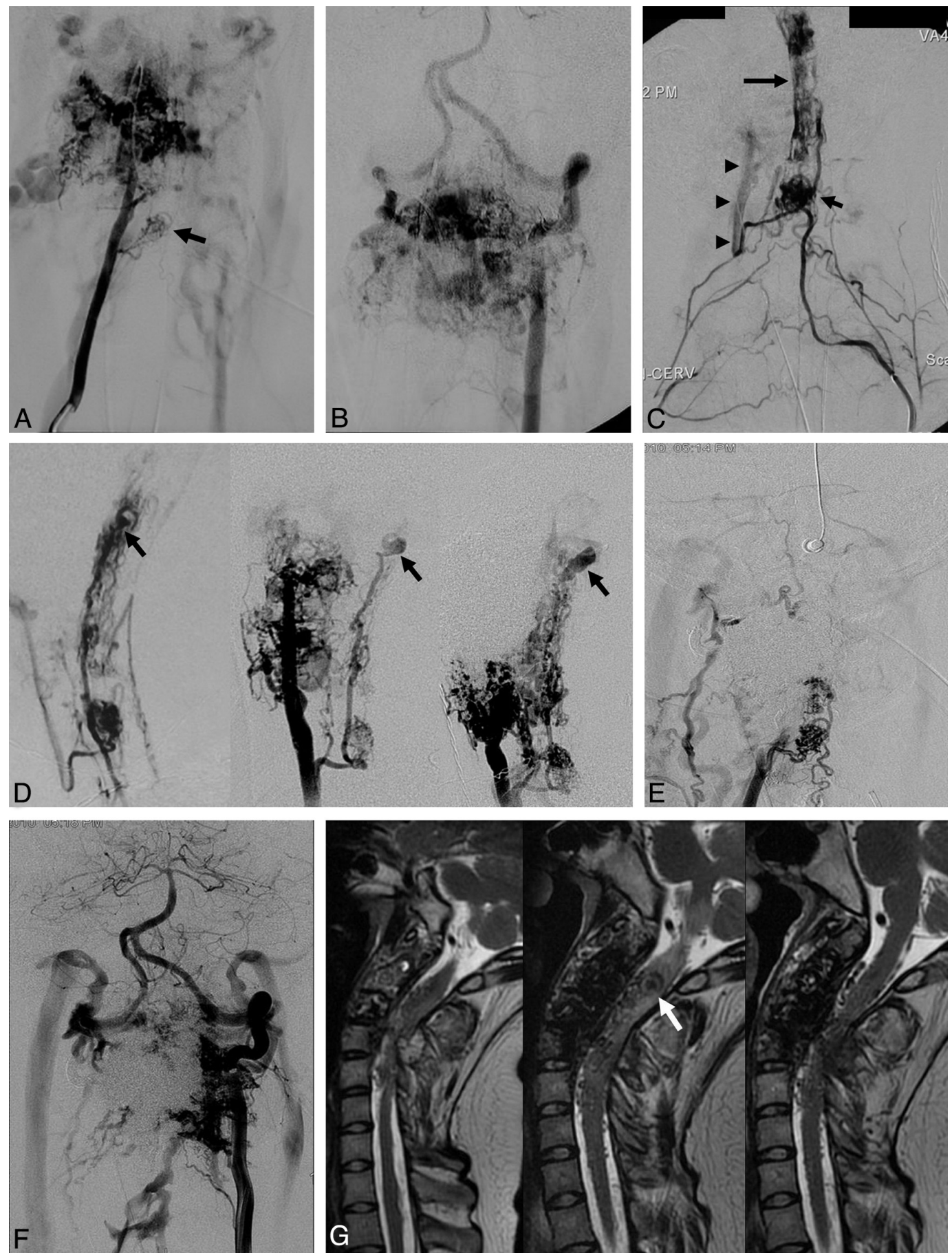

FIG 1. Case 1. A, Right vertebral artery angiogram before the first treatment demonstrates an intramedullary nidus at the C5 level (arrow) and paravertebral/vertebral fistulas at the $C 2$ and $C 3$ levels. B, Left vertebral artery angiogram demonstrates paravertebral/vertebral fistulas at the $\mathrm{C} 2$ and C3 levels. The distal right vertebral artery is opacified in a retrograde fashion due to steal phenomenon. C, Left dorsocervical artery angiogram at the time of the second treatment demonstrates a radiculomedullary artery opacifying the C5 level nidus (short arrow) as well as a second discrete nidus at the C2 and C3 levels (long arrow). There is anastomotic visualization of the right vertebral artery (arrowheads) (compare with A). D, Series of angiographic appearances of the spinal cord AVMs in the lateral view. Right: At the time of the second treatment (lateral view of C) showing 2 discrete nidi. There is a small ASA aneurysm (arrow). Middle: Right vertebral artery injection 21 months later showing enlargement of the ASA aneurysm (arrow). Left: Right vertebral artery angiogram 18 months later from the middle when the patient developed acute left-sided weakness showing further enlargement of the ASA aneurysm (arrow). The distal right vertebral artery is occluded by previous Onyx embolization. E, Right vertebral artery angiogram after the last (eighth) embolization shows minimal residual paravertebral/vertebral fistulas. Spinal cord AVMs are persistently opacified. F, Left vertebral artery angiogram after the last (eighth) embolization shows residual paravertebral/vertebral fistulas. G, Serial T2-weighted sagittal MR images of the cervical spine. Left: After the fourth $n$-BCA embolization showing resolution of T2 signal abnormality seen before the treatment (not shown). Middle: Before the seventh embolization after development of acute left-sided weakness. There is increased $\mathrm{T} 2$ high-signal abnormality involving the upper cervical cord and medulla surrounding the aneurysm (arrow). Signal voids obscuring the $C 2$ and $C 3$ vertebral bodies are due to Onyx. Left: Three months after $n$-BCA embolization of the anterior spinal artery aneurysm, showing disappearance of the aneurysm and resolution of the T2 high-signal abnormality. ASA indicates anterior spinal artery. 

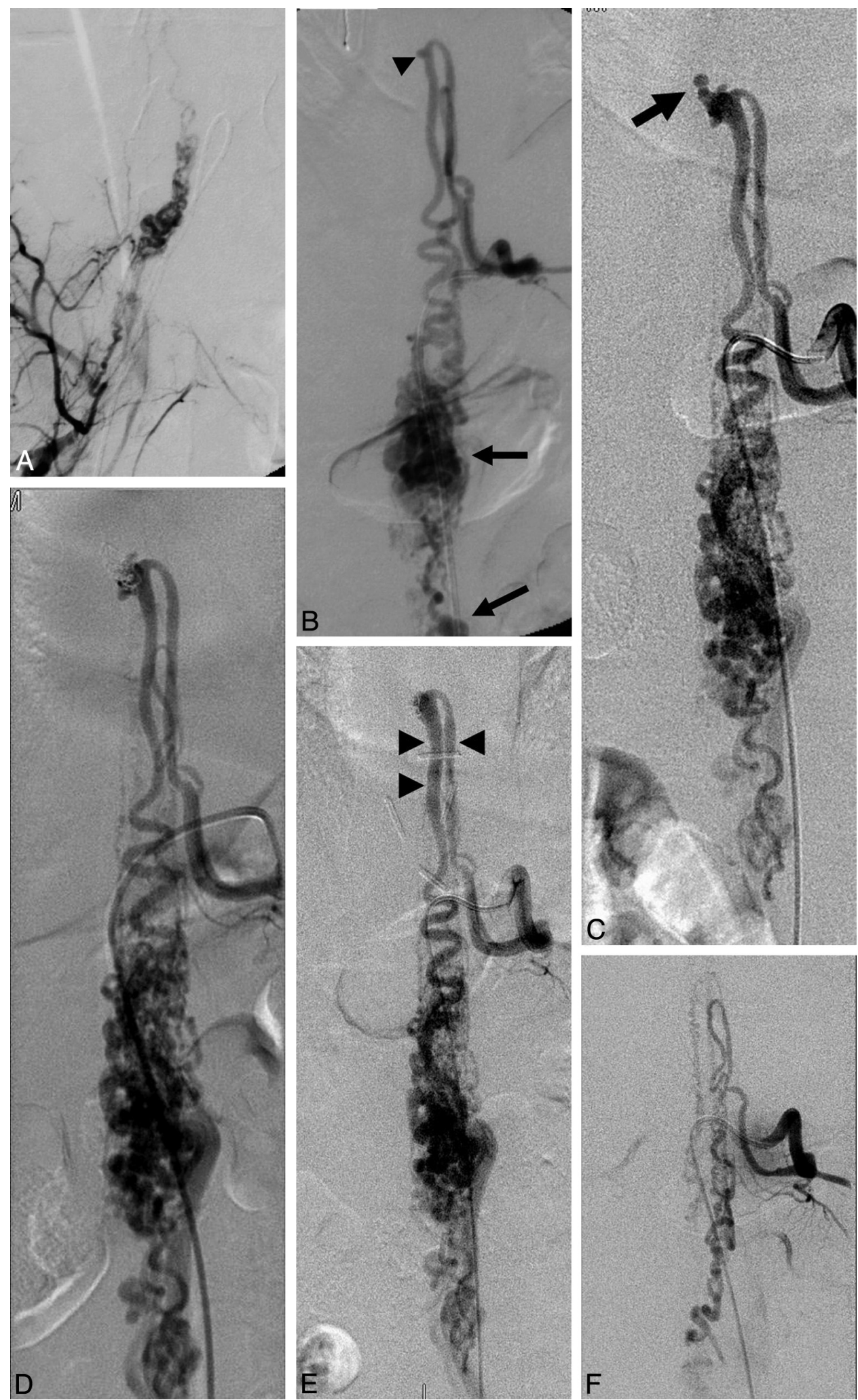

FIG 2. Case 2. A, Right internal iliac artery angiogram in 1995 at the time of presentation demonstrates $\mathrm{S} 2$ radicular arteriovenous shunt draining to the perimedullary veins. B, Left T10 intercostal artery angiogram in 1995 demonstrates 2 nidi of spinal cord AVMs (arrows) at the lower spinal cord and conus supplied by the ASA and the left PSA. There is a small ASA aneurysm (arrowhead). C, Left T10 intercostal artery angiogram in 2004 after the patient developed recurrent SAH demonstrates enlargement of the ASA aneurysm with a pseudoaneurysm at the tip (arrow). The AVM at the conus is fistulous and is supplied by the ASA. The left PSA feeder is smaller than that in 1995. D, Left T10 intercostal artery angiogram after coil embolization of the aneurysm demonstrates occlusion of most of the aneurysm, particularly the pseudoaneurysm. E, Left T10 intercostal artery angiogram in 2007 shows remodeling and progressive occlusion of the ASA aneurysm. There is increased caliber irregularity of the radiculomedullary artery and the ASA (arrowheads). F, Left T10 intercostal artery angiogram in 2011 shows spontaneous occlusion of the ASA. The size of the left PSA has increased. The ASA is partially reconstituted by the right PSA (not shown). ASA indicates anterior spinal artery; PSA, posterior spinal artery. 
or hematomyelia. The intradural lesions associated with SAMS were mostly nidus-type SCAVMs, which is probably one of the reasons for the higher incidence of hemorrhage for patients with SAMS compared with non-SAMS patients. Non-SAMS patients include those with a significant number of microfistulas that usually present with nonhemorrhagic progressive neurologic deficits in adults. Another explanation for the higher hemorrhage rate in SAMS supported by our experience is that intradural lesions of SAMS tend to develop a new aneurysm or enlargement of a preexisting aneurysm. This observation also demonstrates the more dynamic nature of the SAMS compared with non-SAMS lesions, which is well-illustrated in the representative cases. Hemorrhagic episodes after the initial endovascular treatment occurred in 5 patients $(22 \%)$ during follow-up, which was also higher than those in the non-SAMS population.

Nonhemorrhagic neurologic deterioration was the second most common clinical presentation. Acute thrombosis of the draining vein can cause acute neurologic deterioration often associated with pain, clinically mimicking hemorrhage. Venous ectasias or arterial aneurysms may cause mass effect and compressive spinal cord syndromes. Subacute or chronic deterioration of spinal cord function with progressive myelopathy is also common and may be secondary to venous hypertension of the spinal cord. An additional less frequent acute-to-subacute presentation is painful radiculopathy due to nerve root arteriovenous malformations. These lesions are postulated to cause pain due to compression of the nerve root by dilated venous structures in the neural foramen or from a steal phenomenon due to high-flow arteriovenous shunting. Careful assessment of the symptoms and correlation of these with the angioarchitecture of the lesions are the guiding principles for targeted embolization of these complex lesions. For this purpose, assessment of any shared venous drainage of the extra- and intradural portions of the lesion is also important. Symptoms in one patient may be initially attributable to the intramedullary compartment and later due to the extramedullary disease and vice versa, as demonstrated in case 1 .

This longitudinal series of patients with SAMS demonstrates the progressive nature of the disease and poor long-term functional prognosis. However, this series also shows that these complex lesions can be treated safely by endovascular techniques with a palliative strategy focused on preventing hemorrhage, preserving spinal cord function, and relieving pain. Angiographic cure is the exception and only a realistic goal for limited lesions without significant intramedullary involvement. ${ }^{9}$ Even then, we believe that we can improve the natural history of this disease by performing target palliative embolization as illustrated in the representative cases. To maximize the effect of palliative treatment, periodic angiographic examination with intent to treat is important to prevent neurologic deterioration. Diagnostic cross-sectional imaging with CT or MR imaging is currently not sensitive enough to detect development or enlargement of spinal aneurysms of the anterior spinal artery or posterior spinal artery or within the nidus of the AVM. Our recent protocol for follow-up of clinically stable patients is yearly MR imaging without and with contrast administration and clinical examination, and spinal angiography with intent to treat every 3-5 years. If MR imaging changes or clinical deterioration occurs, we perform spinal angiography with intent to treat without delay. For those who have extensive bony or paraspinal lesions, CT is also a useful follow-up tool to assess the extent of bony destruction.

Regarding the technique of embolization, it is most important to close the lesion itself, such as an aneurysm, a fistula site, or a nidus, to obtain a long-lasting effect. Proximal feeder occlusion tends to result in an incomplete transient effect due to collateralization. $n$-BCA is the best embolic agent with a long-lasting occlusive effect for intradural lesions in our experience. Embolization can be safely performed in the anterior spinal artery, ${ }^{10}$ but the safety margin is further increased when posterior spinal artery access to the malformation is feasible. We use neurophysiologic monitoring and superselective provocative testing to add an extra safety margin before embolization. This testing has been demonstrated to have a very high negative predictive value. ${ }^{6}$ Introduction of the Onyx liquid embolic agent has added a useful adjunct in the treatment of SAMS. We have been successful in using this material to treat extensive high-flow extradural fistulas to reduce spinal cord venous hypertension or mass effect or to control bone destruction. Onyx is a suitable embolic agent for this purpose because of its ability to be injected in a large amount from 1 pedicle for a long time. However, poor visualization of the remnant lesion due to permanent high radiopacity of tantalum mixed in Onyx sometimes may complicate future treatments. We have avoided using Onyx in the intradural compartment due to the previously reported potential toxicity of Onyx/dimethylsulfoxide to neural and vascular tissue. ${ }^{11,12}$ In addition, the so called "plug and push" technique used to make Onyx penetrate deep into the lesion often requires significant reflux into the feeding pedicle, which is not feasible for the spinal cord arteries.

\section{CONCLUSIONS}

SAMS is more common than previously reported. Symptoms are mostly caused by the associated intradural vascular malformations but can also be due to extradural lesions. Careful clinical assessment and correlation with angioarchitecture is important for treatment planning. SCAVMs associated with SAMS have a higher tendency to cause hemorrhage compared with non-SAMS lesions. Long-term functional prognosis of SAMS is poor, but it can be improved by targeted palliative embolization. Long-term clinical follow-up and periodic spinal angiographic examinations with intent to treat are important to control this dynamic disease.

\section{REFERENCES}

1. Aminoff MJ, Logue V. The prognosis of patients with spinal vascular malformations. Brain 1974;97:211-18

2. Matsumaru Y, Pongpech S, Laothamas J, et al. Multifocal and metameric spinal cord arteriovenous malformations: review of 19 cases. Interv Neuroradiol 1999;5:27-34

3. Spetzler RF, Detwiler PW, Riina HA, et al. Modified classification of spinal cord vascular lesions. J Neurosurg 2002;96:145-56

4. Bhattacharya JJ, Luo CB, Suh DC, et al. Wyburn-Mason or BonnetDechaume-Blanc as cerebrofacial arteriovenous metameric syndromes (CAMS): A new concept and a new classification. Interv Neuroradiol 2001;7:5-17

5. Berenstein A, Lasjaunias P, ter Brugge K. Spinal arteriovenous malformations. In: Berenstein A, Lasjaunias P, ter Brugge K. Surgical Neuroangiography. 2nd ed. Berlin, Germany: Springer-Verlag; 2004:737-847 
6. Niimi Y, Sala F, Deletis V, et al. Neurophysiologic monitoring and pharmacologic provocative testing for embolization of spinal cord arteriovenous malformations. AJNR Am J Neuroradiol 2004;25:1131-38

7. Couly G, Coltey P, Eichmann A, et al. The angiogenic potentials of the cephalic mesoderm and the origin of brain and head blood vessels. Mech Dev 1995;53:97-112

8. Rodesch G, Hurth M, Alvarez H, et al. Classification of spinal cord arteriovenous shunts: proposal for a reappraisal-the Bicetre experience with 155 consecutive patients treated between 1981 and 1999. Neurosurgery 2002;51:379-80

9. Rodesch G, Hurth M, Alvarez H, et al. Spinal cord intradural arteriovenous fistulae: anatomic, clinical, and therapeutic considerations in a series of 32 consecutive patients seen between 1981 and
2000 with emphasis on endovascular therapy. Neurosurgery 2005; 57:973-83, discussion 973-83

10. Rodesch G, Hurth M, Alvarez H, et al. Embolisation of spinal cord arteriovenous malformations with glue through the anterior spinal axis: review of 20 cases. Interv Neuroradiol 1997;3:131-43

11. Chaloupka JC, Huddle DC, Alderman J, et al. A reexamination of the angiotoxicity of superselective injection of DMSO in the swine rete embolization model. AJNR Am J Neuroradiol 1999;20:401-10

12. Amiridze N, Darwish R. Hemodynamic instability during treatment of intracranial dural arteriovenous fistula and carotid cavernous fistula with Onyx: preliminary results and anesthesia considerations. J Neurointerv Surg 2009;1:146-50 